\title{
Combined pedicled antropyloroplasty and gastric pull-up reconstruction for corrosive esophagogastric stricture
}

Tzu-Ping Chen, MD, Chi-Hsiao Yeh, MD, PhD, Yi-Cheng Wu, MD, and Hui-Ping Liu, MD, Keelung, Taiwan

Supplemental material is available online.

\section{Clinical Summary}

A 40-year-old woman with a history of depression was treated at our hospital for esophageal stricture resulting from ingestion of an alkaline substance. Endoscopic examination revealed a circumferential stricture $22 \mathrm{~cm}$ from the incisors, extending to the distal esophagus. A feeding jejunostomy was created for initial nutritional support. In the 3 months after the injury, the patient received esophageal balloon dilatation on 3 occasions. Contrast meal studies revealed long-segment stricture from the carina to the esopha-

From the Division of Thoracic and Cardiovascular Surgery, Chang Gung Memorial Hospital, Keelung, Taiwan.

Received for publication Jan 30, 2007; accepted for publication Feb 7, 2007.

Address for reprints: Dr. Tzu-Ping Chen, Division of Thoracic and Cardiovascular Surgery, Chang Gung Memorial Hospital, 222 Mai-Cin Road, Keelung 204, Taiwan (E-mail: kk13490@yahoo.com.tw).

J Thorac Cardiovasc Surg 2007;133:1669-70

$0022-5223 / \$ 32.00$

Copyright (C) 2007 by The American Association for Thoracic Surgery doi:10.1016/j.jtcvs.2007.02.006 gogastic junction and distal antrum resulting

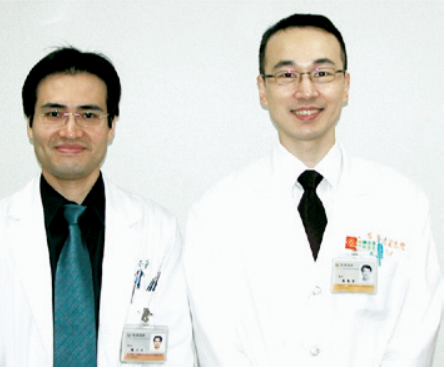

Drs Chen and Yeh (left to right) in partial gastric outlet obstruction (Figure 1). After failure of endoscopic dilatation, she was subsequently scheduled for surgery owing to progressive vomiting and swallowing difficulty.

Esophagectomy was performed via video-assisted thoracic surgery with the patient under general anesthesia. An upper midline laparotomy was then performed. The gastrocolic and gastrosplenic ligaments were dissected, and the short gastric vessels were divided. The hepatogastric ligament was then opened, and the left gastric artery and vein were doubly ligated and cut. After a Kocher maneuver, the stomach was fully mobilized and transected between the points of three fourths of the lesser curvature and the lateral gastroesophageal junction with 3 Autosuture staples (GIA75; Ethicon Endosurgery, Cincinnati, Ohio). On closer inspection, there was fibrotic scarring in the distorted region of antrum/pylorus, which was contracted and rigid. Palpation of this area revealed $5-\mathrm{cm}$ segmental thickening in the gastric outlet region. The lesser curvature was divided at one third from the pylorus, and a $5 \times 5-\mathrm{cm}$ pedicled flap was created based on the right gastric artery (Figure 2). After the scarred antrum/pylorus was opened longitudinally, the gastric flap was attached to the antrotomy with a double-layer hand-sewn approximation (Figures 2 and E1). The staple line along the lesser curvature was oversewn with a continuous layer of absorbable suture. Esopha-
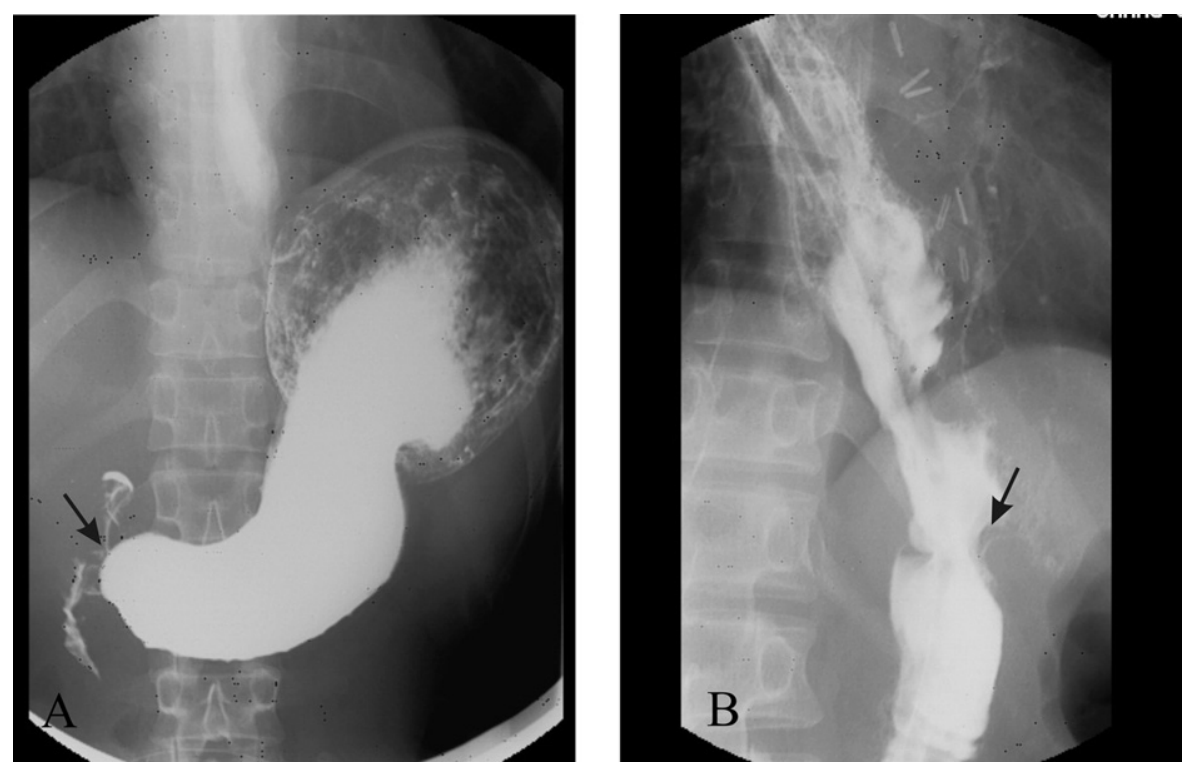

Figure 1. A, Preoperative upper gastrointestinal series revealed stricture with ulcer crater formation at the distal antrum leading to partial gastric outlet obstruction (black arrow). B, Postoperative esophagography revealed smooth passage of contrast media with patent anastomosis. 

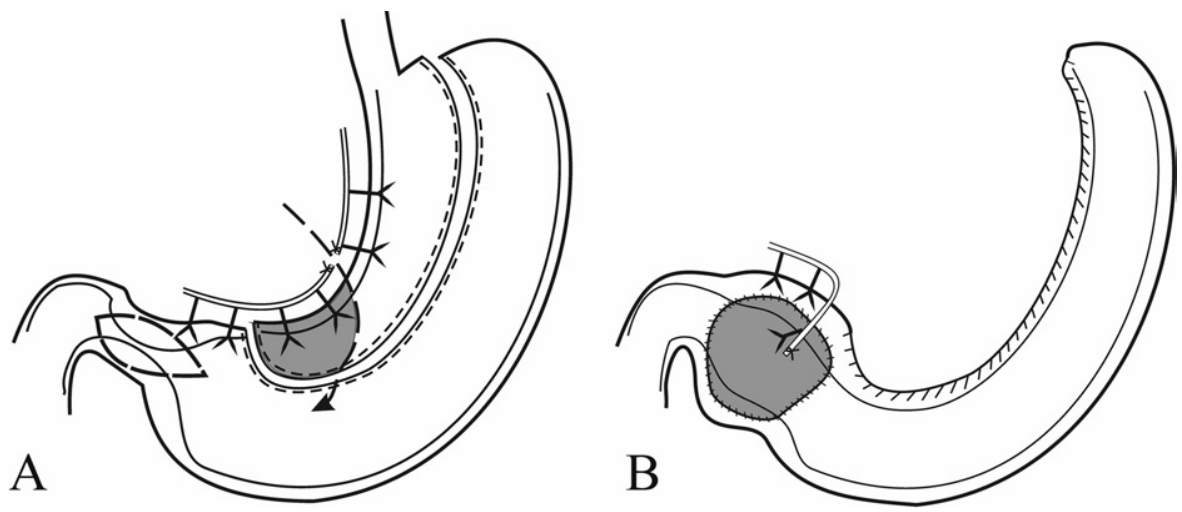

Figure 2. Schematic illustration of gastric flap creation. A, Gastroplasty with staples: one third of the lesser curvature is excised for creation of a pedicle flap (preserving right gastric artery integrity). B, Pedicle gastric flap coverage on the opened antrum/pylorus region.

gogastrostomy was accomplished by gastric pull-up of the left side of the neck through the posterior mediastinal route. Subsequent contrast studies revealed a patent cervical anastomosis and adequate pyloric drainage (Figure 1). The patient was able to resume solid and liquid oral intake from postoperative day 9. At 24 months' follow-up, satisfactory body weight gain was noted.

\section{Discussion}

Corrosive gastric outlet obstruction is generally considered an uncommon entity, with an estimated $20 \%$ concomitant incidence of corrosive esophagitis. Associated esophageal strictures have been reported in $6 \%$ to $63 \%$ of patients with corrosive gastric injury. ${ }^{1}$ For asymptomatic esophageal stricture with gastric outlet obstruction, the gastric lesion remains hidden until the stricture is solved endoscopically. Patients with severe and extensive esophageal strictures, resistant to dilatations, require esophageal reconstruction. Concomitant gastric outlet obstruction caused by antral stricture may complicate surgery when the distorted region is extensive.

Traditionally, the colon is the preferable conduit for esophageal replacement in light of the low associated morbidity and anatomic features, including constant blood supply and length allowing for extension to the neck. ${ }^{2}$ Gupta $^{3}$ has proposed augmentation gastroduodenoplasty using a longitudinal split colon for brunt contracted stomach. Other investigators, however, have advocated using the stomach as the conduit because of its dependable blood supply, richness of the submucosal vascular network, and the fact that repositioning of the stomach requires only one anastomosis. ${ }^{4}$ In cases in which long-segment stenosis of the antrum/pylorus is apparent, pyloric drainage through pyloroplasty is not possible. Bilroth I subtotal gastrectomy may interfere with gastric tube length. Here we have described a modified technique of simulta- neous antropyloroplasty and gastric pull-up reconstruction in a patient with mild-to-moderate gastric burn. The right gastric artery does not contribute to gastric tube blood flow ${ }^{5}$ but does offer a superior supply to the pedicled flap. Thus, adequate volume can be maintained for gastroplasty. This procedure is easy to perform and preserves the integrity of cicatrized stomach. Adequate widening of the lumen can be achieved with minimal anatomic and physiologic destruction. We also prefer use of the stomach as it shortens operating time and provides the safest and most physiologic replacement.

In conclusion, pedicled flap antropyloroplasty is an alternative surgical option for corrosive antral stricture. It can effectively widen the narrowed antrum and restore gastric tube length span for subsequent esophageal reconstruction.

\section{References}

1. Chaudhary A, Puri AS, Dhar P, Reddy P, Sachdev A, Lahoti D, et al. Elective surgery for corrosive-induced gastric injury. World J Surg. 1996;20:703-6.

2. Han Y, Cheng QS, Li XF, Wang XP. Surgical management of esophageal strictures after caustic burns: a 30 years of experience. World $J$ Gastroenterol. 2004;10:1846-9.

3. Gupta S. Surgical management of corrosive strictures following acid burns of upper gastrointestinal tract. Eur J Cardiothorac Surg. 1996; 10:934-40.

4. Zhou LH, Jiang YG, Wang RW, Lin YD, Gong TQ, Zhao YP, et al. Management of corrosive esophageal burn in 149 cases. $J$ Thorac Cardiovasc Surg. 2005;130:449-55.

5. Tabira Y, Sakaguchi T, Kuhara H, Teshima K, Tanaka M, Kawasuji M. The width of a gastric tube has no impact on outcome after esophagectomy. Am J Surg. 2004;187:417-21. 

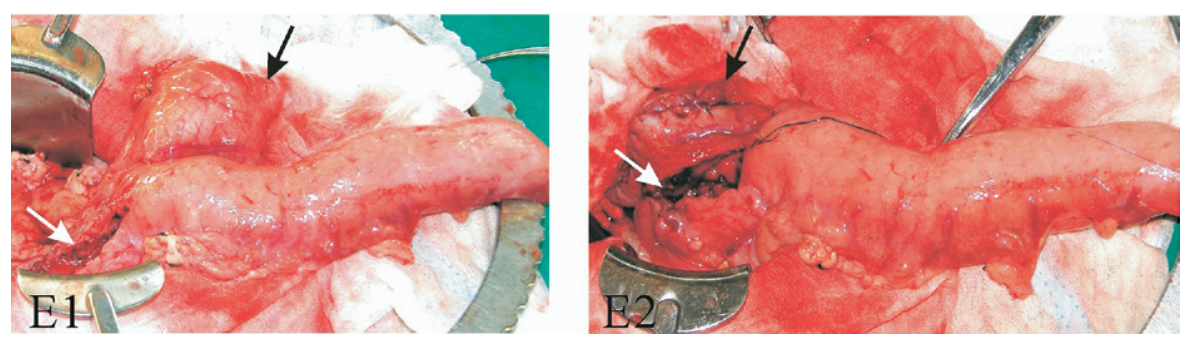

Figure E1. Operative view of the flap (black arrow) divided from the lesser curvature. A 6-cm incision was made through the distal antrum (white arrow). E2, Final anastomosis of the anterior wall gastric conduit (white arrow). 